\title{
On the optical theorem and optimal extinction, scattering and absorption in lossy media
}

\author{
Sven Nordebo*, Mats Gustafsson ${ }^{\dagger}$, Yevhen Ivanenko* \\ *Department of Physics and Electrical Engineering, Linnæus University, 35195 Växjö, Sweden. E-mail: sven.nordebo@lnu.se \\ ${ }^{\dagger}$ Department of Electrical and Information Technology, Lund University, Box 118, 22100 Lund, Sweden.
}

\begin{abstract}
This paper reformulates and extends some recent analytical results concerning a new optical theorem and the associated physical bounds on absorption in lossy media. The analysis is valid for any linear scatterer (such as an antenna), consisting of arbitrary materials (bianisotropic, etc.) and arbitrary geometries, as long as the scatterer is circumscribed by a spherical volume embedded in a lossy background medium. The corresponding formulas are here reformulated and extended to encompass magnetic as well as dielectric background media. Explicit derivations, formulas and discussions are also given for the corresponding bounds on scattering and extinction. A numerical example concerning the optimal microwave absorption and scattering in atmospheric oxygen in the $60 \mathrm{GHz}$ communication band is included to illustrate the theory.
\end{abstract}

Index Terms-optical theorem, extinction, scattering, absorption, absorbing media.

\section{INTRODUCTION}

Perhaps counter-intuitive, the presence of external losses in the surrounding medium implies major difficulties in the formulation of an optical theorem for a general scattering object, and a reasonably simple analytical solution is typically available only for spheres [1], [2], [3], [4], see also [5], [6], [7]. Analytical solutions based on the spherical vector wave expansion (Mie theory) [3] have recently been used to formulate an optical theorem and to derive explicit formulas for the optimal multipole absorption, scattering and extinction of a spherical object embedded in a lossy medium [5], [6]. In [7] has been shown that the theory developed in [5] is valid not only for the individual multipole absorption of a rotationally invariant sphere, but also for the absorption of an arbitrary scatterer (consisting of arbitrary materials and structures) embedded inside a spherical inclusion, and furthermore that the bound is valid also as a summation over all multipole fields (the total field) as long as the losses in the surrounding medium are not vanishing. In this contribution, we reformulate and extend the analytical results given in [7] to encompass magnetic as well as dielectric background media, and we give explicit derivations and formulas also for the corresponding bounds on scattering and extinction. In essence, the theory gives new fundamental bounds on absorption, scattering and extinction that limits any single frequency super resolution effects for a scatterer in a lossy surrounding medium, with possible applications e.g., with antennas and resonators in communications [8], [9], [10], [11], [12], [13], plasmonics and metamaterials [14], [15], [16] and medicine [17], [18], [19].

\section{OPTICAL THEOREM FOR A SPHERICAL INCLUSION IN LOSSY MEDIA}

\section{A. Notation and conventions}

The electric and magnetic field intensities $\boldsymbol{E}$ and $\boldsymbol{H}$ are given in SI-units [20] and the time convention for time harmonic fields (phasors) is given by $\mathrm{e}^{-\mathrm{i} \omega t}$, where $\omega$ is the angular frequency and $t$ the time. Let $\mu_{0}, \epsilon_{0}, \eta_{0}$ and $c_{0}$ denote the permeability, the permittivity, the wave impedance and the speed of light in vacuum, respectively, and where $\eta_{0}=\sqrt{\mu_{0} / \epsilon_{0}}$ and $c_{0}=1 / \sqrt{\mu_{0} \epsilon_{0}}$. The wave number of vacuum is given by $k_{0}=\omega \sqrt{\mu_{0} \epsilon_{0}}$, and hence $\omega \mu_{0}=k_{0} \eta_{0}$ and $\omega \epsilon_{0}=k_{0} \eta_{0}^{-1}$. The definition of the spherical vector waves [21] and their most important properties employed in this paper can be found in [5, $\mathrm{A}(1)-\mathrm{A}(32)]$. Here, $l=1,2, \ldots$, denotes the multipole order, $m=-l, \ldots, l$, the azimuthal index and $\tau=1$ indicates a transverse electric (TE) magnetic multipole and $\tau=2$ a transverse magnetic (TM) electric multipole, respectively. The regular spherical Bessel functions, the Neumann functions, the spherical Hankel functions of the first kind and the corresponding Riccati-Bessel functions [21] are denoted $\mathrm{j}_{l}(z), \mathrm{y}_{l}(z), \mathrm{h}_{l}^{(1)}(z)=\mathrm{j}_{l}(z)+\mathrm{iy}_{l}(z), \psi_{l}(z)=z \mathrm{j}_{l}(z)$ and $\xi_{l}(z)=z \mathrm{~h}_{l}^{(1)}(z)$, respectively, all of order $l$. The real and imaginary parts and the complex conjugate of a complex number $\zeta$ are denoted by $\operatorname{Re}\{\zeta\}, \operatorname{Im}\{\zeta\}$ and $\zeta^{*}$, respectively.

\section{B. Absorption, scattering and extinction}

Consider a scattering problem where an absorbing structure (such as a receiving antenna) is embedded in an infinite homogeneous and isotropic background medium having relative permeability $\mu$, relative permittivity $\epsilon$, wave impedance $\eta_{0} \eta$ where $\eta=\sqrt{\mu / \epsilon}$ and wave number $k=k_{0} \sqrt{\mu \epsilon}$. The absorbing structure is made of an arbitrary material (general bianisotropic etc.) in arbitrary geometry, and is circumscribed by a minimal sphere of radius $a$. The scattering body $V$ is considered to be the whole circumscribing sphere with surface denoted $\partial V$. The background medium is passive and in general lossy, with complex-valued parameters satisfying $\operatorname{Im}\{\mu\} \geq 0$, $\operatorname{Im}\{\epsilon\} \geq 0$ and $\operatorname{Im}\{k\} \geq 0$.

Let the total fields outside $V$ be decomposed in incident and scattered fields as $\boldsymbol{E}=\boldsymbol{E}_{\mathrm{i}}+\boldsymbol{E}_{\mathrm{s}}$ and $\boldsymbol{H}=\boldsymbol{H}_{\mathrm{i}}+\boldsymbol{H}_{\mathrm{s}}$, respectively. Following [22], [5], [7] the optical theorem can now be derived from the power balance at the surface $\partial V$

$$
P_{\mathrm{a}}=-P_{\mathrm{s}}+P_{\mathrm{t}}+P_{\mathrm{i}},
$$


where $P_{\mathrm{a}}, P_{\mathrm{s}}, P_{\mathrm{t}}$ and $P_{\mathrm{i}}$ are the absorbed, scattered, extinct (total) and the absorbed incident powers, respectively, defined by

$$
\begin{aligned}
& P_{\mathrm{a}}=-\frac{1}{2} \operatorname{Re}\left\{\int_{\partial V} \boldsymbol{E} \times \boldsymbol{H}^{*} \cdot \hat{\boldsymbol{n}} \mathrm{d} S\right\}, \\
& P_{\mathrm{s}}=\frac{1}{2} \operatorname{Re}\left\{\int_{\partial V} \boldsymbol{E}_{\mathrm{s}} \times \boldsymbol{H}_{\mathrm{s}}^{*} \cdot \hat{\boldsymbol{n}} \mathrm{d} S\right\}, \\
& P_{\mathrm{t}}=-\frac{1}{2} \operatorname{Re}\left\{\int_{\partial V}\left(\boldsymbol{E}_{\mathrm{i}} \times \boldsymbol{H}_{\mathrm{s}}^{*}+\boldsymbol{E}_{\mathrm{s}} \times \boldsymbol{H}_{\mathrm{i}}^{*}\right) \cdot \hat{\boldsymbol{n}} \mathrm{d} S\right\}, \\
& P_{\mathrm{i}}=-\frac{1}{2} \operatorname{Re}\left\{\int_{\partial V} \boldsymbol{E}_{\mathrm{i}} \times \boldsymbol{H}_{\mathrm{i}}^{*} \cdot \hat{\boldsymbol{n}} \mathrm{d} S\right\},
\end{aligned}
$$

and where the surface integrals are defined with an outward unit normal $\hat{\boldsymbol{n}}$.

Let $a_{\tau m l}^{\mathrm{i}}$ and $f_{\tau m l}$ denote the multipole coefficients of the incident (regular) and the scattered (outgoing) spherical vector waves, respectively, as defined in [5, $\mathrm{A}(1)]$. Based on the orthogonality of the spherical vector waves on the spherical surface $\partial V$ as given by [5, $\mathrm{A}(31)$ and $\mathrm{A}(32)]$, it can be shown that

$$
\begin{aligned}
P_{\mathrm{s}} & =\frac{\operatorname{Re}\{1 / \eta\}}{2|k|^{2} \eta_{0}} \sum_{\tau, m, l} A_{\tau l}\left|f_{\tau m l}\right|^{2}, \\
P_{\mathrm{t}} & =\frac{\operatorname{Re}\{1 / \eta\}}{2|k|^{2} \eta_{0}} \sum_{\tau, m, l} 2 \operatorname{Re}\left\{B_{\tau l} a_{\tau m l}^{\mathrm{i} *} f_{\tau m l}\right\}, \\
P_{\mathrm{i}} & =\frac{\operatorname{Re}\{1 / \eta\}}{2|k|^{2} \eta_{0}} \sum_{\tau, m, l} C_{\tau l}\left|a_{\tau m l}^{\mathrm{i}}\right|^{2},
\end{aligned}
$$

where

$$
\begin{aligned}
& A_{\tau l}=\frac{1}{\operatorname{Re}\{1 / \eta\}} \begin{cases}-\operatorname{Im}\left\{\xi_{l} \xi_{l}^{\prime *} / \eta^{*}\right\} & \tau=1, \\
\operatorname{Im}\left\{\xi_{l}^{\prime} \xi_{l}^{*} / \eta^{*}\right\} & \tau=2,\end{cases} \\
& B_{\tau l}=\frac{1}{2 \operatorname{RiRe}\{1 / \eta\}}\left\{\begin{array}{cc}
\xi_{l} \psi_{l}^{\prime *} / \eta^{*}-\psi_{l}^{*} \xi_{l}^{\prime} / \eta & \tau=1, \\
-\xi_{l}^{\prime} \psi_{l}^{*} / \eta^{*}+\psi_{l}^{\prime *} \xi_{l} / \eta & \tau=2,
\end{array}\right. \\
& C_{\tau l}=\frac{1}{\operatorname{Re}\{1 / \eta\}}\left\{\begin{array}{cc}
\operatorname{Im}\left\{\psi_{l} \psi_{l}^{\prime *} / \eta^{*}\right\} & \tau=1, \\
-\operatorname{Im}\left\{\psi_{l}^{\prime} \psi_{l}^{*} / \eta^{*}\right\} & \tau=2,
\end{array}\right.
\end{aligned}
$$

for $\tau=1,2$ and $l=1, \ldots, \infty$, and where the arguments of the Riccati-Bessel functions are $z=k a$. Notice the modification in the definition of these parameters in terms of the relative wave impedance $\eta$ (due to the presence of the magnetic property), in comparison to [5, Eqs. (9) through (11)] and [7. Eqs. (37) through (42)]. Similar as in [5], [7], it can be readily shown that $A_{\tau l}>0, C_{\tau l} \geq 0$ and it is noticed that $B_{\tau l}$ is in general a complex-valued constant. As expected, it is possible to show that (9) through (11) are invariant to the change $\mu \leftrightarrow \epsilon$ under the summation over $\tau=1,2$. For a lossless medium ( $k$ and $\eta$ are real-valued), it can furthermore be shown that $A_{\tau l}=1, B_{\tau l}=-1 / 2$ and $C_{\tau l}=0$, see [5, p. 3], in agreement with e.g., [21, Eq. (7.18)].

\section{OPTIMAL CROSS SECTIONS}

\section{A. Optimal absorption}

The optimal absorption presented here has previously been derived in [7, Eqs. (43) through (51)]. The approach is briefly summarized below to provide a framework for a more general setting including scattering and extinction, as well as to incorporate both electric and magnetic surrounding media.

Consider the power absorbed from a single partial wave with fixed multi-index $n=(\tau, m, l)$,

$$
\begin{array}{r}
P_{\mathrm{a}, n}=\frac{\operatorname{Re}\{1 / \eta\}}{2|k|^{2} \eta_{0}}\left(-A_{\tau l}\left|f_{n}\right|^{2}+2 \operatorname{Re}\left\{B_{\tau l} a_{n}^{\mathrm{i} *} f_{n}\right\}\right. \\
\left.+C_{\tau l}\left|a_{n}^{\mathrm{i}}\right|^{2}\right),
\end{array}
$$

where we have employed the optical theorem (1) as well as (6) through (8). Let the scattering coefficients $f_{n}$ be given by the T-matrix [21, Eq. (7.34)] for an arbitrary linear scatterer inside the spherical surface $\partial V$, so that

$$
f_{n}=\sum_{n^{\prime}} T_{n, n^{\prime}} a_{n^{\prime}}^{\mathrm{i}} .
$$

It is observed that $(12)$ is a concave function of the complexvalued T-matrix elements $T_{n, n^{\prime}}$ with respect to the primed index $n^{\prime}$. Hence, we can employ the following complex derivative (for fixed $n$ )

$$
\frac{\partial}{\partial T_{n, n^{\prime}}}=a_{n^{\prime}}^{\mathrm{i}} \frac{\partial}{\partial f_{n}},
$$

to find the stationarity condition

$$
a_{n^{\prime}}^{\mathrm{i}} \sum_{n^{\prime \prime}} a_{n^{\prime \prime}}^{\mathrm{i} *} T_{n, n^{\prime \prime}}^{*}=\frac{B_{\tau l}}{A_{\tau l}} a_{n}^{\mathrm{i} *} a_{n^{\prime}}^{\mathrm{i}},
$$

which is an infinite-dimensional linear system of equations in $T_{n, n^{\prime \prime}}$ for a given $n$, see also [7, Eqs. (43) through (45)]. For a truncated (finite-dimensional) partial wave expansion the unique minimum norm (pseudo-inverse) solution to (15) is given by

$$
T_{n, n^{\prime}}^{\mathrm{opt}}=\frac{B_{\tau l}^{*}}{A_{\tau l}\left\|\boldsymbol{a}^{\mathrm{i}}\right\|^{2}} a_{n}^{\mathrm{i}} a_{n^{\prime}}^{\mathrm{i} *},
$$

where $\left\|\boldsymbol{a}^{\mathrm{i}}\right\|^{2}=\sum_{n}\left|a_{n}^{\mathrm{i}}\right|^{2}$. It is noted that the unique pseudoinverse in 16 can be extended by adding a sequence from the corresponding null space as

$$
T_{n, n^{\prime}}^{\mathrm{opt}} \rightarrow T_{n, n^{\prime}}^{\mathrm{opt}}+t_{n^{\prime}} \quad \text { where } \quad \sum_{n^{\prime}} a_{n^{\prime}}^{\mathrm{i}} t_{n^{\prime}}=0
$$

and which hence satisfies the stationarity condition 15 and corresponds to the same scattering coefficient $f_{n}$ as in (13).

By completing the squares based on (12), 13, and (16, it can be shown that

$$
\begin{gathered}
P_{\mathrm{a}, n}=\frac{\operatorname{Re}\{1 / \eta\}}{2|k|^{2} \eta_{0}}\left(-A_{\tau l}\left|\sum_{n^{\prime}}\left(T_{n, n^{\prime}}-\frac{B_{\tau l}^{*}}{A_{\tau l}\left\|\boldsymbol{a}^{\mathrm{i}}\right\|^{2}} a_{n}^{\mathrm{i}} a_{n^{\prime}}^{\mathrm{i} *}\right) a_{n^{\prime}}^{\mathrm{i}}\right|^{2}\right. \\
\left.+\left(\frac{\left|B_{\tau l}\right|^{2}}{A_{\tau l}}+C_{\tau l}\right)\left|a_{n}^{\mathrm{i}}\right|^{2}\right)
\end{gathered}
$$

From the concavity of this expression $\left(A_{\tau l}>0\right)$, it follows that the last term gives the optimal absorption

$$
P_{\mathrm{a}, n}^{\mathrm{opt}}=\frac{\operatorname{Re}\{1 / \eta\}}{2|k|^{2} \eta_{0}}\left(\frac{\left|B_{\tau l}\right|^{2}}{A_{\tau l}}+C_{\tau l}\right)\left|a_{n}^{\mathrm{i}}\right|^{2} .
$$


Consider now a plane wave $\boldsymbol{E}_{\mathrm{i}}(\boldsymbol{r})=\boldsymbol{E}_{0} \mathrm{e}^{\mathrm{i} k \hat{\boldsymbol{k}} \cdot \boldsymbol{r}}$ with vector amplitude $\boldsymbol{E}_{0}$, propagation direction $\hat{\boldsymbol{k}}$ and power intensity (at $\boldsymbol{r}=\mathbf{0}) I_{\mathrm{i}}=\left|\boldsymbol{E}_{0}\right|^{2} \operatorname{Re}\left\{1 / 2 \eta_{0} \eta\right\}$. The corresponding multipole expansion coefficients are given by $a_{\tau m l}^{\mathrm{i}}=4 \pi \mathrm{i}^{l-\tau+1} \boldsymbol{E}_{0}$. $\mathbf{A}_{\tau m l}^{*}(\hat{\boldsymbol{k}})$, where $\mathbf{A}_{\tau m l}(\hat{\boldsymbol{k}})$ are the vector spherical harmonics, see [5, Eqs. (5) and (A4)]. It can also be shown that

$$
\sum_{m=-l}^{l}\left|a_{\tau m l}^{\mathrm{i}}\right|^{2}=2 \pi(2 l+1)\left|\boldsymbol{E}_{0}\right|^{2},
$$

for $\tau=1,2$, see e.g., [5, Eqs. (6)]. The optimal normalized absorption cross section is now obtained as

$$
\begin{aligned}
Q_{\mathrm{a}}^{\mathrm{opt}}= & \frac{1}{\pi a^{2} I_{\mathrm{i}}} \sum_{n} P_{\mathrm{a}, n}^{\mathrm{opt}} \\
& =\frac{2}{|k a|^{2}} \sum_{\tau=1}^{2} \sum_{l=1}^{\infty}(2 l+1)\left(\frac{\left|B_{\tau l}\right|^{2}}{A_{\tau l}}+C_{\tau l}\right) .
\end{aligned}
$$

In [7, Eqs. (53) through (57)] is shown that the coefficients $A_{\tau l}, B_{\tau l}$ and $C_{\tau l}$ have factorial increase, are bounded and have factorial decrease, respectively, and hence that the expansion in 21) converges whenever there are nonzero losses in the background medium with $\operatorname{Im}\{k\}>0$. In the lossless case (with $A_{\tau l}=1, B_{\tau l}=-1 / 2$ and $C_{\tau l}=0$ ) we obtain the divergent series

$$
Q_{\mathrm{a}, L}^{\mathrm{opt}}=\frac{1}{2(k a)^{2}} \sum_{\tau=1}^{2} \sum_{l=1}^{L}(2 l+1)=\frac{1}{(k a)^{2}} L(L+2),
$$

indicating the optimal absorption for each multipole order $l$, see also [5, Eqs. (19) and (20)].

\section{B. Optimal scattering}

The scattered power of a partial wave with fixed multi-index $n=(\tau, m, l)$ is given by

$$
P_{\mathrm{s}, n}=\frac{\operatorname{Re}\{1 / \eta\}}{2|k|^{2} \eta_{0}} A_{\tau l}\left|f_{n}\right|^{2},
$$

$c f$., 6. Consider now the constrained optimization problem

$$
\begin{array}{ll}
\text { maximize } & P_{\mathrm{s}, n} \\
\text { subject to } & P_{\mathrm{a}, n} \geq 0,
\end{array}
$$

where $P_{\mathrm{a}, n}$ is the corresponding absorbed power given by (12). This is a problem of maximizing a convex function over a convex set, which can be formulated equivalently as

$$
\begin{aligned}
\operatorname{maximize} & \left|f_{n}\right|^{2} \\
\text { subject to } & A_{\tau l}\left|f_{n}\right|^{2}-2 \operatorname{Re}\left\{B_{\tau l} a_{n}^{\mathrm{i} *} f_{n}\right\} \\
& -C_{\tau l}\left|a_{n}^{\mathrm{i}}\right|^{2}=0,
\end{aligned}
$$

where it is a priori known that the constraint must be active. Here, $f_{n}$ is given by (13) and the optimization is with respect to the T-matrix elements $T_{n, n^{\prime}}$ for a fixed multi-index $n$.

The Lagrangian for this problem is

$$
\begin{array}{r}
L=\left|f_{n}\right|^{2}+\alpha\left(A_{\tau l}\left|f_{n}\right|^{2}-B_{\tau l} a_{n}^{\mathrm{i} *} f_{n}-B_{\tau l}^{*} a_{n}^{\mathrm{i}} f_{n}^{*}\right. \\
\left.-C_{\tau l}\left|a_{n}^{\mathrm{i}}\right|^{2}\right),
\end{array}
$$

where $\alpha$ is the Lagrange multiplier. The condition for stationarity is obtained by applying the complex derivative (14) yielding

$$
a_{n^{\prime}}^{\mathrm{i}} \sum_{n^{\prime \prime}} a_{n^{\prime \prime}}^{\mathrm{i} *} T_{n, n^{\prime \prime}}^{*}=\frac{\alpha B_{\tau l}}{1+\alpha A_{\tau l}} a_{n}^{\mathrm{i} *} a_{n^{\prime}}^{\mathrm{i}}
$$

with the unique minimum norm (pseudo-inverse) solution

$$
T_{n, n^{\prime}}^{\mathrm{opt}}=\frac{\beta B_{\tau l}^{*}}{\left\|\boldsymbol{a}^{\mathrm{i}}\right\|^{2}} a_{n}^{\mathrm{i}} a_{n^{\prime}}^{\mathrm{i} *},
$$

where

$$
\beta=\frac{\alpha}{1+\alpha A_{\tau l}} .
$$

The parameter $\beta$ (for fixed $n$ ) is determined by inserting (13) into the constraint equation in 25 and completing the squares based on 28. The following quadratic condition appears after some labor

$$
\begin{aligned}
& A_{\tau l} \mid \sum_{n^{\prime}}\left(T_{n, n^{\prime}}\right.\left.-\frac{\beta B_{\tau l}^{*}}{\left\|\boldsymbol{a}^{\mathrm{i}}\right\|^{2}} a_{n}^{\mathrm{i}} a_{n^{\prime}}^{\mathrm{i} *}\right)\left.a_{n^{\prime}}^{\mathrm{i}}\right|^{2} \\
&+\left(A_{\tau l} \beta-1\right) 2 \operatorname{Re}\left\{B_{\tau l} a_{n}^{\mathrm{i} *} \sum_{n^{\prime}} T_{n, n^{\prime}} a_{n^{\prime}}^{\mathrm{i}}\right\} \\
&-\left(\beta^{2} A_{\tau l}\left|B_{\tau l}\right|^{2}+C_{\tau l}\right)\left|a_{n}^{\mathrm{i}}\right|^{2}=0 .
\end{aligned}
$$

It is seen that the condition 30 invariant to the choice of null space solutions as expressed in (17). Hence, by inserting (28) into (30) the following quadratic equation is obtained

$$
\beta^{2}-2 \beta \frac{1}{A_{\tau l}}-\frac{C_{\tau l}}{A_{\tau l}\left|B_{\tau l}\right|^{2}}=0,
$$

yielding the solution

$$
\beta_{\tau l}=\frac{1}{A_{\tau l}}+\sqrt{\frac{1}{A_{\tau l}^{2}}+\frac{C_{\tau l}}{A_{\tau l}\left|B_{\tau l}\right|^{2}}},
$$

where it is the larger root that yields the maximally scattered power. The corresponding optimal scattering coefficient is obtained by inserting (28) into (13) yielding

$$
f_{n}^{\mathrm{opt}}=\beta_{\tau l} B_{\tau l}^{*} a_{n}^{\mathrm{i}}
$$

The optimal normalized scattering cross section can now be obtained as

$$
\begin{aligned}
& Q_{\mathrm{s}}^{\mathrm{opt}}=\frac{1}{\pi a^{2} I_{\mathrm{i}}} \sum_{n} P_{\mathrm{s}, n}^{\mathrm{opt}} \\
& \quad=\frac{2}{|k a|^{2}} \sum_{\tau=1}^{2} \sum_{l=1}^{\infty}(2 l+1) \beta_{\tau l}^{2} A_{\tau l}\left|B_{\tau l}\right|^{2},
\end{aligned}
$$

where (23), 33 and 20) have been used. Based on the asymptotic behavior of the coefficients $A_{\tau l}, B_{\tau l}$ and $C_{\tau l}$, it is readily seen that the expansion in 34 converges whenever there are nonzero losses in the background medium with $\operatorname{Im}\{k\}>0, c f$., [7, Eqs. (53) through (57)]. In the lossless case (with $A_{\tau l}=1, B_{\tau l}=-1 / 2$ and $C_{\tau l}=0$ ) we obtain the divergent series $Q_{\mathrm{s}, L}^{\mathrm{opt}}=4 L(L+2) /\left(k_{0} a\right)^{2}$, similar as in 22). 


\section{Optimal extinction}

The optimal extinction can be obtained similarly to the case with optimal scattering as described above. The extinct (total) power of a partial wave with fixed multi-index $n=(\tau, m, l)$ is given by

$$
P_{\mathrm{t}, n}=\frac{\operatorname{Re}\{1 / \eta\}}{2|k|^{2} \eta_{0}} 2 \operatorname{Re}\left\{B_{\tau l} a_{n}^{\mathrm{i} *} f_{n}\right\},
$$

$c f .$, 77. Consider now the constrained optimization problem

$$
\begin{array}{ll}
\text { maximize } & P_{\mathrm{t}, n} \\
\text { subject to } & P_{\mathrm{a}, n} \geq 0,
\end{array}
$$

where $P_{\mathrm{a}, n}$ is the corresponding absorbed power given by (12). This is a problem of maximizing a convex (linear) function over a convex set, which can be formulated equivalently as

$$
\begin{array}{ll}
\text { maximize } & 2 \operatorname{Re}\left\{B_{\tau l} a_{n}^{\mathrm{i} *} f_{n}\right\} \\
\text { subject to } & A_{\tau l}\left|f_{n}\right|^{2}-2 \operatorname{Re}\left\{B_{\tau l} a_{n}^{\mathrm{i} *} f_{n}\right\} \\
& -C_{\tau l}\left|a_{n}^{\mathrm{i}}\right|^{2}=0,
\end{array}
$$

and where the constraint is a priori known to be active. Again, $f_{n}$ is given by (13) and the optimization is with respect to the T-matrix elements $T_{n, n^{\prime}}$ for a fixed multi-index $n$.

The Lagrangian for this problem is

$$
\begin{aligned}
& L=B_{\tau l} a_{n}^{\mathrm{i} *} f_{n}+B_{\tau l}^{*} a_{n}^{\mathrm{i}} f_{n}^{*} \\
& +\alpha\left(A_{\tau l}\left|f_{n}\right|^{2}-B_{\tau l} a_{n}^{\mathrm{i} *} f_{n}-B_{\tau l}^{*} a_{n}^{\mathrm{i}} f_{n}^{*}-C_{\tau l}\left|a_{n}^{\mathrm{i}}\right|^{2}\right),
\end{aligned}
$$

where $\alpha$ is the Lagrange multiplier. The condition for stationarity is obtained by applying the complex derivative 14 yielding

$$
a_{n^{\prime}}^{\mathrm{i}} \sum_{n^{\prime \prime}} a_{n^{\prime \prime}}^{\mathrm{i} *} T_{n, n^{\prime \prime}}^{*}=\frac{\alpha-1}{\alpha} \frac{B_{\tau l}}{A_{\tau l}} a_{n}^{\mathrm{i} *} a_{n^{\prime}}^{\mathrm{i}}
$$

with the unique minimum norm (pseudo-inverse) solution

$$
T_{n, n^{\prime}}^{\mathrm{opt}}=\frac{\beta B_{\tau l}^{*}}{\left\|\boldsymbol{a}^{\mathrm{i}}\right\|^{2}} a_{n}^{\mathrm{i}} a_{n^{\prime}}^{\mathrm{i} *}
$$

where

$$
\beta=\frac{\alpha-1}{\alpha A_{\tau l}} .
$$

It is observed that the solution (40) is identical to 28 in terms of the parameter $\beta$, and it is merely the relation to $\alpha$ that differs, $c f$., 41) and 29]. This means that the optimal solutions in terms of $\beta$ and $f_{n}$ in (32) and (33) are also valid in the case of optimal extinction. The optimal normalized extinction cross section can now be obtained as

$$
Q_{\mathrm{t}}^{\mathrm{opt}}=\frac{1}{\pi a^{2} I_{\mathrm{i}}} \sum_{n} P_{\mathrm{t}, n}^{\mathrm{opt}}=\frac{4}{|k a|^{2}} \sum_{\tau=1}^{2} \sum_{l=1}^{\infty}(2 l+1) \beta_{\tau l}\left|B_{\tau l}\right|^{2},
$$

where (35), (33) and (20) have been used. Based on the asymptotic behavior of the coefficients $A_{\tau l}, B_{\tau l}$ and $C_{\tau l}$, it is readily seen that the expansion in (42) converges whenever there are nonzero losses in the background medium with $\operatorname{Im}\{k\}>0, c f$., [7, Eqs. (53) through (57)]. In the lossless case (with $A_{\tau l}=1, B_{\tau l}=-1 / 2$ and $C_{\tau l}=0$ ) we obtain the divergent series $Q_{\mathrm{t}, L}^{\mathrm{opt}}=4 L(L+2) /\left(k_{0} a\right)^{2}$, similar as in 22).

\section{NUMERICAL EXAMPLES}

As a numerical example we consider the atmospheric absorption by oxygen in the $60 \mathrm{GHz}$ band [23], [24], [25], which is of great importance due to its capability to mitigate interference in short range communication systems [8], [9], [10]. As an additional interesting property of the lossy atmosphere, we demonstrate below that it also ultimately limits any super resolution capabilities of small antennas.

In Fig. 1 is shown the absorption coefficient $\alpha=N \sigma_{\mathrm{a}}$ of air (plotted in $\mathrm{dB} / \mathrm{km}$ ) where $N$ is the number density of the absorptive gas (oxygen) and $\sigma_{\mathrm{a}}$ the absorption cross section per molecule. The data is taken from the open source database HITRAN (high-resolution transmission molecular absorption database) [26]. Due to the magnetic dipole moment of oxygen and its related rotational transitions, the background medium is magnetic with $\alpha=2 k_{0} \operatorname{Im}\{\sqrt{\mu}\}$ and permeability $\mu=1+\mathrm{i} \mu^{\prime \prime}$ where $\mu^{\prime \prime}=\alpha / k_{0}$, assuming that $\mu^{\prime \prime}$ is small. In the numerical examples below we have used $\mu^{\prime \prime}=2 \cdot 10^{-6}$ corresponding approximately to the peak absorption seen in Fig. 1

In Figs. 2 and 3 are shown the optimal normalized absorption and scattering cross sections $Q_{\mathrm{a}}^{\mathrm{opt}}$ and $Q_{\mathrm{s}}^{\mathrm{opt}}$ given by (21) and (34), respectively. The graphs are calculated for a lossy background medium with $\epsilon=1$ and $\mu=1+\mathrm{i} 2 \cdot 10^{-6}$ (atmospheric oxygen), the electrical sizes $k_{0} a=1,10,100$ (corresponding to $a=0.08,0.8,8 \mathrm{~cm}$ ) and maximal multipole order $L=1, \ldots, 150$ where $l \leq L$. Finally, in Fig. 4 we illustrate that the constraints on absorption from the lossy atmosphere are comparable to those imposed on the antenna effective area by a surface resistivity of $R_{\mathrm{s}}=10^{-4} \Omega / \square$ on a spherical shell, $c f$., [13, Fig. 3 on p. 5285]. In practice, this means that it would be meaningless to design a "super conducting" antenna at $60 \mathrm{GHz}$ with a surface resistivity smaller than $R_{\mathrm{s}}=10^{-4} \Omega / \square$, see also [13, Fig. 7 on p. 5287].

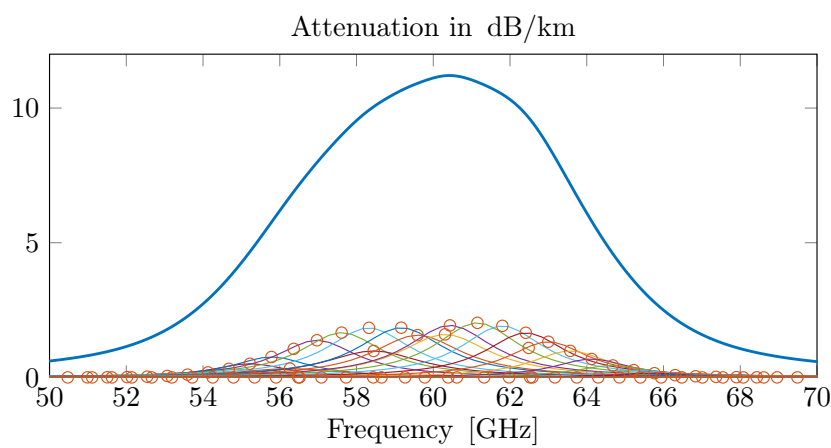

Fig. 1. Absorption coefficient of dry air at normal pressure in the $60 \mathrm{GHz}$ oxygen band (blue solid line). The solid lines indicated with an 'o' show the pressure broadened Lorentz profiles for each individual magnetic dipole transition of oxygen at normal pressure in air.

\section{CONCLUSION}

In this contribution we have reformulated and extended some recent analytical results concerning an optical theorem and the associated optimal extinction, scattering and absorption in lossy surrounding media. In essence, the theory gives new fundamental bounds that limits any single frequency super 


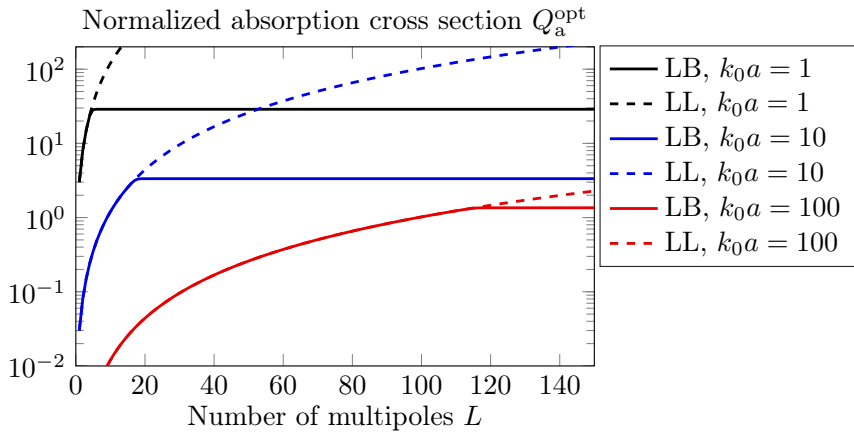

Fig. 2. Optimal normalized absorption cross section $Q_{\mathrm{a}}^{\mathrm{opt}}$ as a function of maximal multipole order $L$, and a lossy background (LB) atmosphere with $\mu^{\prime \prime}=2 \cdot 10^{-6}$. The dashed lines show the optimal absorption cross section for the lossless case (LL) $Q_{\mathrm{a}, L}^{\mathrm{opt}}=L(L+2) /\left(k_{0} a\right)^{2}$.

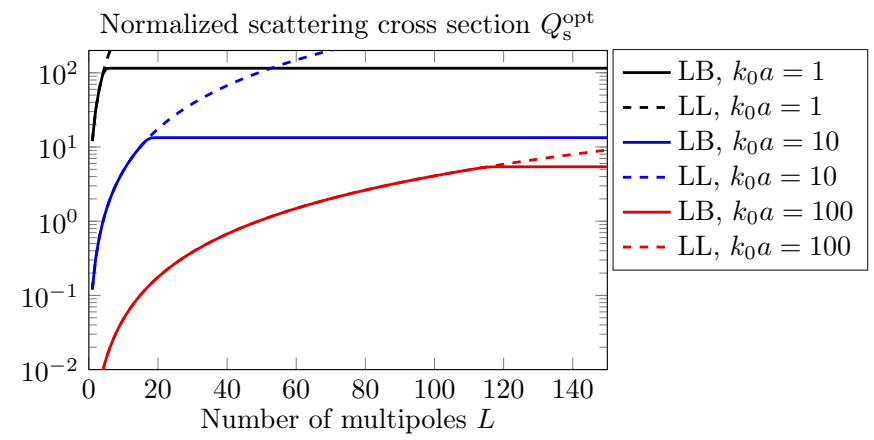

Fig. 3. Optimal normalized scattering cross section $Q_{\mathrm{s}}^{\mathrm{opt}}$ as a function of maximal multipole order $L$, and a lossy background (LB) atmosphere with $\mu^{\prime \prime}=2 \cdot 10^{-6}$. The dashed lines show the optimal scattering cross section for the lossless case (LL) $Q_{\mathrm{s}, L}^{\mathrm{opt}}=4 L(L+2) /\left(k_{0} a\right)^{2}$.

resolution capabilities for a scatterer in a lossy surrounding media, with possible applications e.g., with antennas and resonators in communications, plasmonics and medicin.

\section{REFERENCES}

[1] C. F. Bohren and D. P. Gilra, "Extinction by a spherical particle in an absorbing medium," J. Colloid Interface Sci., vol. 72, no. 2, pp. 215221, 1979.

[2] A. Lebedev, M. Gartz, U. Kreibig, and O. Stenzel, "Optical extinction by spherical particles in an absorbing medium: Application to composite absorbing films," Eur. Phys. J. D., vol. 6, no. 3, pp. 365-373, 1999.

[3] I. W. Sudiarta and P. Chylek, "Mie-scattering formalism for spherical particles embedded in an absorbing medium," J. Opt. Soc. Am. A, vol. 18, no. 6, pp. 1275-1278, 2001

[4] S. Durant, O. Calvo-Perez, N. Vukadinovic, and J.-J. Greffet, "Light scattering by a random distribution of particles embedded in absorbing media: diagrammatic expansion of the extinction coefficient," J. Opt. Soc. Am. A, vol. 24, no. 9, pp. 2943-2952, 2007.

[5] S. Nordebo, G. Kristensson, M. Mirmoosa, and S. Tretyakov, "Optimal plasmonic multipole resonances of a sphere in lossy media," Phys. Rev. $B$, vol. 99, no. 5, p. 054301, 2019.

[6] S. Nordebo, M. Mirmoosa, and S. Tretyakov, "On the quasistatic optimal plasmonic resonances in lossy media," J. Appl. Phys., vol. 125, p. 103105, 2019.

[7] Y. Ivanenko, M. Gustafsson, and S. Nordebo., "Optical theorems and physical bounds on absorption in lossy media," Opt. Express, vol. 27, no. 23, pp. 34 323-34342, 2019.

[8] C. Park and T. S. Rappaport, "Short-range wireless communications for next-generation networks: UWB, $60 \mathrm{GHz}$ millimeter-wave WPAN, and ZigBee," IEEE Wireless Communications, pp. 70-78, 2007.
Normalized absorption cross section $Q^{\text {opt }}$

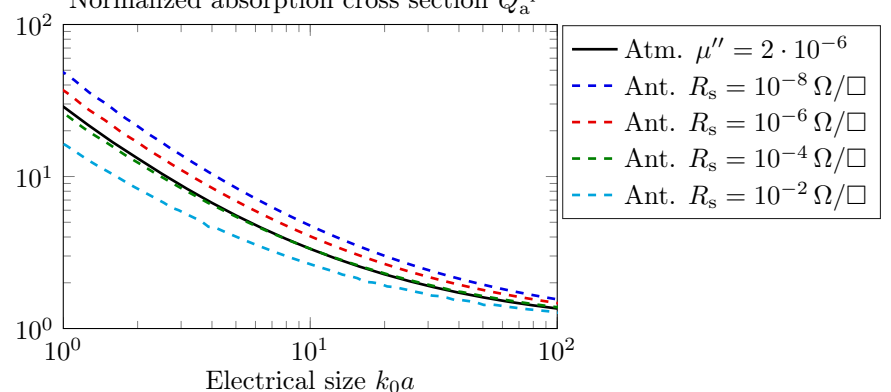

Fig. 4. Comparison of the optimal absorption cross section $Q_{\mathrm{a}}^{\mathrm{opt}} 21$ for the lossy atmosphere (Atm.) with $\mu^{\prime \prime}=2 \cdot 10^{-6}$, and the maximum effective area for an externally tuned spherical shell (Ant.) with surface resistivity $R_{\mathrm{S}}=$ $10^{-8}, 10^{-6}, 10^{-4}, 10^{-2} \Omega / \square$ and a lossless atmosphere [13].

[9] N. D. Hawkins, R. Steele, D. C. Rickard, and C. R. Shepherd, "Path loss characteristics of $60 \mathrm{GHz}$ transmissions," Elect. Lett., vol. 21, no. 22 pp. 1054-1055, 1985.

[10] J. Wang, H. Zhang, T. Lv, and T. A. Gulliver, "Capacity of $60 \mathrm{GHz}$ wireless communication systems over fading channels," Journal of networks, vol. 7, no. 1, pp. 203-209, 2012.

[11] A. Karlsson, "Physical limitations of antennas in a lossy medium," IEEE Trans. Antennas Propagat., vol. 52, pp. 2027-2033, 2004.

[12] A. K. Skrivervik, M. Bosiljevac, and Z. Sipus, "Fundamental limits for implanted antennas: Maximum power density reaching free space," IEEE Trans. Antennas Propagat., vol. 67, no. 8, pp. 4978-4988, 2019.

13] M. Gustafsson and M. Capek, "Maximum gain, effective area, and directivity," IEEE Trans. Antennas Propagat., vol. 67, no. 8, pp. 52825293, 2019.

[14] S. A. Maier, Plasmonics: Fundamentals and Applications. Berlin: Springer-Verlag, 2007.

[15] S. I. Maslovski, C. R. Simovski, and S. A. Tretyakov, "Overcoming black body radiation limit in free space: metamaterial superemitter," New J. Phys., vol. 18, p. 013034, 2016.

[16] C. A. Valagiannopoulos and S. A. Tretyakov, "Theoretical concepts of unlimited-power reflectors, absorbers, and emitters with conjugately matched layers," Phys. Rev. B, vol. 94, p. 125117, 2016.

[17] X. Huang, P. K. Jain, I. H. El-Sayed, and M. A. El-Sayed, "Plasmonic photothermal therapy (PPTT) using gold nanoparticles," Lasers Med Sci, vol. 23, pp. 217-228, 2008.

[18] E. Sassaroli, K. C. P. Li, and B. E. O’Neil, "Radio frequency absorption in gold nanoparticle suspensions: a phenomenological study," J. Phys. D: Appl. Phys., vol. 45, pp. 1-15, 2012, 075303.

[19] S. Nordebo, M. Dalarsson, Y. Ivanenko, D. Sjöberg, and R. Bayford, "On the physical limitations for radio frequency absorption in gold nanoparticle suspensions," J. Phys. D: Appl. Phys., vol. 50, no. 15, p. $155401,2017$.

[20] J. D. Jackson, Classical Electrodynamics, 3rd ed. New York: John Wiley \& Sons, 1999

[21] G. Kristensson, Scattering of Electromagnetic Waves by Obstacles. SciTech Publishing, Edison, NJ, 2016.

[22] C. F. Bohren and D. R. Huffman, Absorption and Scattering of Light by Small Particles. New York: John Wiley \& Sons, 1983

[23] J. H. V. Vleck, "The absorption of microwaves by oxygen," Phys. Rev., vol. 71, no. 7, pp. 413-424, 1947.

[24] M. L. Meeks and A. E. Lilley, "The microwave spectrum of oxygen in the Earth's atmosphere," Journal of Geophysical Research, vol. 68, no. 6, pp. 1683-1703, 1963.

[25] M. Y. Tretyakov, M. A. Koshelev, V. V. Dorovskikh, D. S. Makarov, and P. W. Rosenkranz, "60-GHz oxygen band: precise broadening and central frequencies of fine-structure lines, absolute absorption profile at atmospheric pressure, and revision of mixing coefficients," Journal of Molecular Spectroscopy, vol. 231, pp. 1-14, 2005.

[26] I. E. Gordon and et al, "The HITRAN2016 molecular spectroscopic database," Journal of Quantitative Spectroscopy \& Radiative Transfer vol. 203, pp. 3-69, 2017. 